\title{
WILDFIRE DAMAGE ASSESSMENT USING MULTI-TEMPORAL SENTINEL-2 DATA
}

\author{
M. Chung ${ }^{1}$, M. Jung ${ }^{1}$, Y. Kim ${ }^{1, *}$ \\ ${ }^{1}$ Dept. of Civil and Environmental Engineering, Seoul National University, Seoul, Republic of Korea \\ - (mkjung4876, worldhurrah, yik)@ snu.ac.kr
}

Commission III, ICWG III/IVa

KEY WORDS: Wildfire damage assessment, Multi-temporal image analysis, OBIA, Change detection, Sentinel-2

\begin{abstract}
:
Recently, the drastic climate changes have increased the importance of wildfire monitoring and damage assessment as well as the possibility of wildfire occurrence. Estimation of wildfire damage provides the information on wildfire-induced ecological changes and supports the decision-making process for post-fire treatment activities. For accurate wildfire damage assessment, the discrimination between disaster-induced and natural changes is crucial because they usually coupled together.

In this study, Sentinel-2 images were employed to assess the damage from a wildfire, which occurred in the coniferous forest of Gangneung, Gangwon Province, South Korea on April 2019. The images were captured from both Sentinel-2A and -2B, shortening the temporal interval of available pre- and post-fire images. Multi-temporal image analysis was performed in both object and pixelbased with two commonly used spectral indices, NDVI and NBR. Additional image pair from the same period of 2018 was used to distinguish the fire-affected regions from the naturally changed area and compared with the results from using only one pair of images from 2019. The experimental results showed that the change detection performance could be affected by the number of image pairs and spectral indices used to discriminate burned region from unburned region. Thus it verified the significance of adequately employing annual multi-pair satellite images for wildfire damage assessment.
\end{abstract}

\section{INTRODUCTION}

Due to drastic climate changes in recent decades, the importance of wildfire monitoring and change detection has been growing along with the increased wildfire occurrence possibility (Liu et al., 2010). Wildfire burn scar provides information for estimating financial or ecological damages by the fire and planning of post-fire treatment activities (Lentile et al., 2006). Several previous studies were proposed to verify the suitability of Sentinel-2 data for detecting wildfire burn scars as well as assessing the conditions of vegetation and soil (Huang et al., 2016; Martinis et al., 2017; Navarro et al., 2017). However, those studies were limited to large scale wildfires, which occurred in certain Mediterranean regions (Mallinis et al., 2018) Therefore, in this study, we tried to detect the burn scars of a wildfire, which occurred in the coniferous forest of Gangneung, Gangwon Province in South Korea in April 2019 with Sentinel2 images. It is a rare study case employing Sentinel-2 data for the East Asian region and small scale wildfire, which burned less than 1,000 ha.

As one of the common application fields using multi-temporal remote sensing images, many change detection techniques have been developed in both pixel-based and object-based approaches. Pixel-based algorithms are theoretically simple and known to well perform for low and medium-resolution images (Hussain et al., 2013). However, in the high-resolution images, pixels are not spatially independent, and it makes these conventional pixel-based methods less effective than objectbased methods (Aggarwal et al., 2016).

Compared with pixel-based approaches, object-based image analysis assumes that the landscapes are composed of meaningful objects corresponding to ground entities and patches of surface cover (Blaschke et al., 2000). These object-based approaches are introduced to reduce the noises and the subsequent image processing computations, but it may affect the accuracy of the final image processing results.

The purpose of this study is to enhance the accuracy of the burned area mapping with multi-temporal high-resolution satellite images. In doing so, two commonly used spectral indices (SI), NDVI (Normalized Difference Vegetation Index) and NBR (Normalized Burn Ratio), were employed to estimate the fire-affected area. To address the differences from the image-processing unit, burned area mapping was performed for both object-based and pixel-based image analysis. From evaluation with Sentinel-2 images, SIs showed certain differences in discriminating burned region from unburned region depending on the number of image pairs.

For object-based image analysis (OBIA), one of the superpixel techniques, named SLIC (Simple Linear Iterative Clustering), was chosen for image segmentation. Superpixel techniques group pixels into segments based on their homogeneity with neighbouring pixels. By capturing the redundancy in the image, superpixels reduce the complexity of the image and the complexity of the following image processing tasks (Achanta et al., 2010). Since superpixels can well preserve image edges by image oversegmentation (Neubert, Protzel, 2012), they have considerable potential for improving the accuracy of OBIA. Moreover, as the superpixels generated from the images are merely segments from adjacent pixels with similar colours, additional clustering was applied by DBSCAN (Density-Based Spatial Clustering of Applications with Noise).

This paper is organized as follows. Section 2 illustrates the study site and satellite data used in this study. Section 3 describes the method to estimate the burned area with multitemporal image analysis. The experimental results of fireaffected area mapping are presented in Section 4, and the

\footnotetext{
* Corresponding author
} 
meanings and limitations of the study are addressed as well. As a conclusion, Section 5 sums up the points of the study and suggests future studies.

\section{STUDY SITE AND DATA}

\subsection{Study Site}

The area of interest is a wildfire site from Gangneung, Gangwon Province in South Korea, which suffered severe wildfire in April 2019. Gangneung is located in the east side of the Tae Baek Mountains, which is one of the major mountain ranges in South Korea and features a maritime climate in its highlands. The fire began on April 4th, 2019 and continued for four days, burning 714.8 ha of pure stand pine forests (Korea Forest Service).

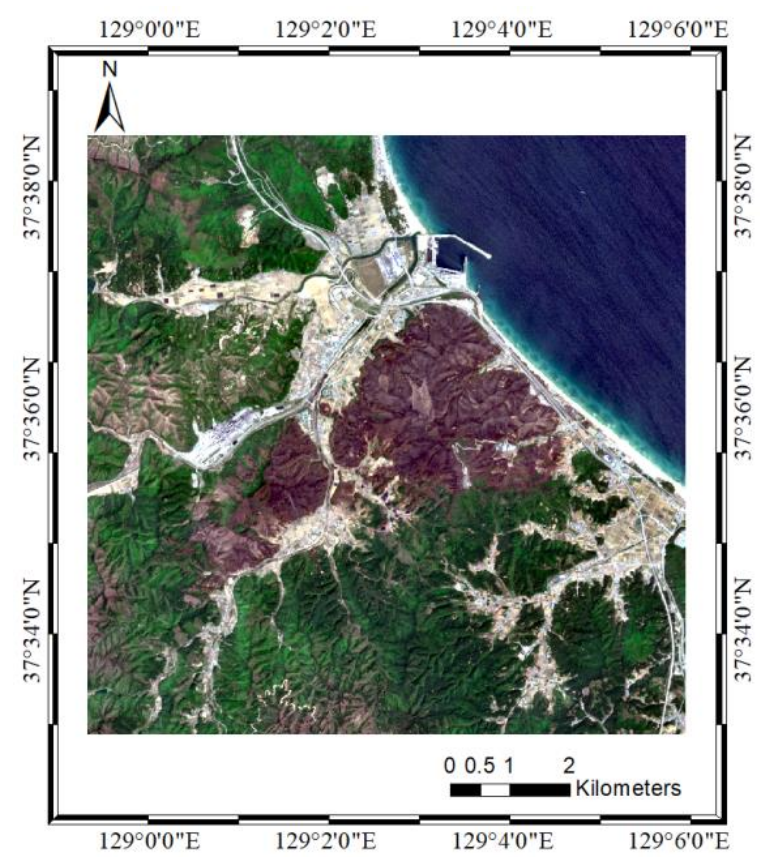

Figure 1. Location of study site (Sentinel-2A image captured after 2019 Gangwon wildfire event)

\subsection{Data}

The data used in this study is from Sentinel-2 (2A and 2B) sensor, which includes 13 VNIR and SWIR bands with a spatial resolution of $10 \mathrm{~m}$ to $60 \mathrm{~m}$ (Table 1 and 2). Two pairs of highresolution images were captured, each pair on 2018 and 2019. Based on the Sentinel-2 data availability of 2019 when the wildfire occurred, the images from 2018 were chosen to have close acquisition date for elimination of phenological changes irrelevant to wildfire events. Each pair of images have about 1month-temporal-interval and obtained as Level-2A product.

\begin{tabular}{|c|c|c|c|}
\hline $\begin{array}{c}\text { Acquisition } \\
\text { date }\end{array}$ & Sensor & Description & Image size \\
\hline $2018 / 03 / 26$ & Sentinel-2A & Pre-fire & \multirow{4}{*}{$\begin{array}{c}1000 \times 1000 \\
\quad \text { (pixels) }\end{array}$} \\
\hline $2018 / 04 / 20$ & Sentinel-2B & Pre-fire & \\
\hline $2019 / 03 / 26$ & Sentinel-2B & Pre-fire & \\
\hline $2019 / 04 / 20$ & Sentinel-2A & Post-fire & \\
\hline
\end{tabular}

Table 1. Specifications of images used in this study

\begin{tabular}{|c|c|c|c|}
\hline $\begin{array}{c}\text { Band number and } \\
\text { name }\end{array}$ & $\begin{array}{c}\text { Central } \\
\text { wavelength } \\
(\mathrm{nm})\end{array}$ & $\begin{array}{c}\text { Bandwidth } \\
(\mathrm{nm})\end{array}$ & $\begin{array}{c}\text { Spatial } \\
\text { resolution } \\
(\mathrm{m})\end{array}$ \\
\hline
\end{tabular}

\begin{tabular}{|c|c|c|c|c|}
\hline B1 & $\begin{array}{c}\text { Coastal } \\
\text { aerosol }\end{array}$ & 443 & 20 & 60 \\
\hline B2 & Blue & 490 & 65 & 10 \\
\hline B3 & Green & 560 & 35 & 10 \\
\hline B4 & Red & 665 & 30 & 10 \\
\hline B5 & Red edge 1 & 705 & 15 & 20 \\
\hline B6 & Red edge 2 & 740 & 15 & 20 \\
\hline B7 & Red edge 3 & 783 & 20 & 20 \\
\hline B8 & NIR & 842 & 115 & 10 \\
\hline B8A & NIR narrow & 865 & 20 & 20 \\
\hline B9 & Water vapour & 945 & 20 & 60 \\
\hline B10 & Circus & 1375 & 30 & 60 \\
\hline B11 & SWIR1 & 1610 & 90 & 20 \\
\hline B12 & SWIR2 & 2190 & 180 & 20 \\
\hline
\end{tabular}

Table 1. Specifications of Sentinel-2 MSI (Multispectral Instrument) sensor (ESA, 2015)

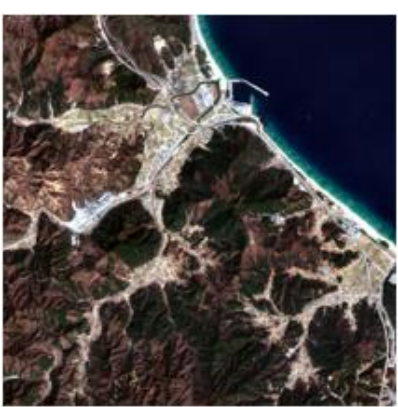

(a)

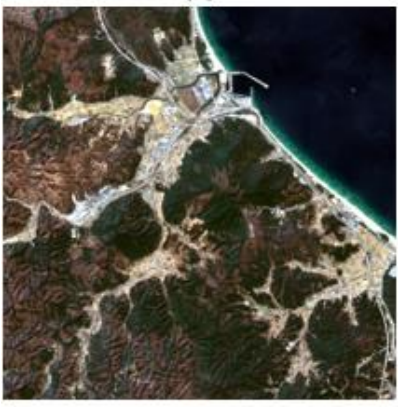

(c)

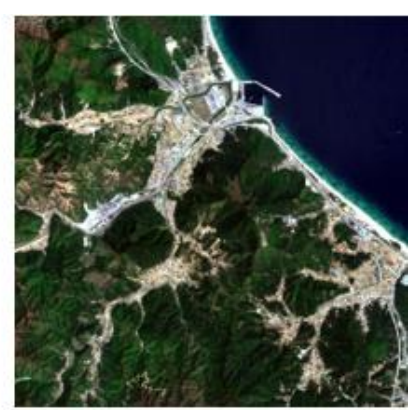

(b)

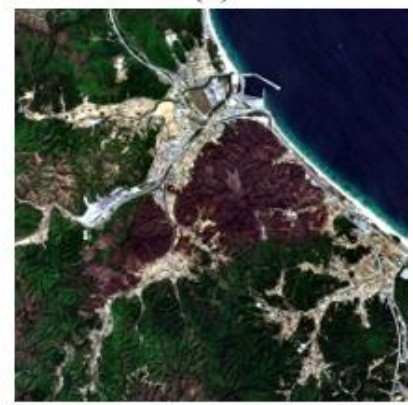

(d)
Figure 2. Sentinel-2 images used in this study; captured on (a) March 26, 2018 (b) April 20, 2018 (c) March 26, 2019 (d) April 20, 2019 (Copernicus Sentinel data 2019, processed by ESA)

\section{METHODOLOGY}

The proposed algorithm is devised to evaluate the change detection performance of object and pixel-based image analysis when applied to wildfire damage assessment with multitemporal high-resolution satellite images. For both object and pixel-based approaches, the burned area was detected by widely used SIs, in this case, NDVI and NBR values with Otsu's thresholding (Otsu, 1979). In OBIA, it requires an additional two steps for image segmentation before change detection step; superpixel generation and superpixel clustering. First, for superpixel generation, RGB images were used as an input to SLIC. Then, additional clustering using DBSCAN was applied to cluster superpixels into a certain size of objects for change detection based on spatial adjacency and colour similarity. The following subsections describe the method for estimating the fire-affected area in detail. 
Fig. 3 shows the overall flowchart of the study.

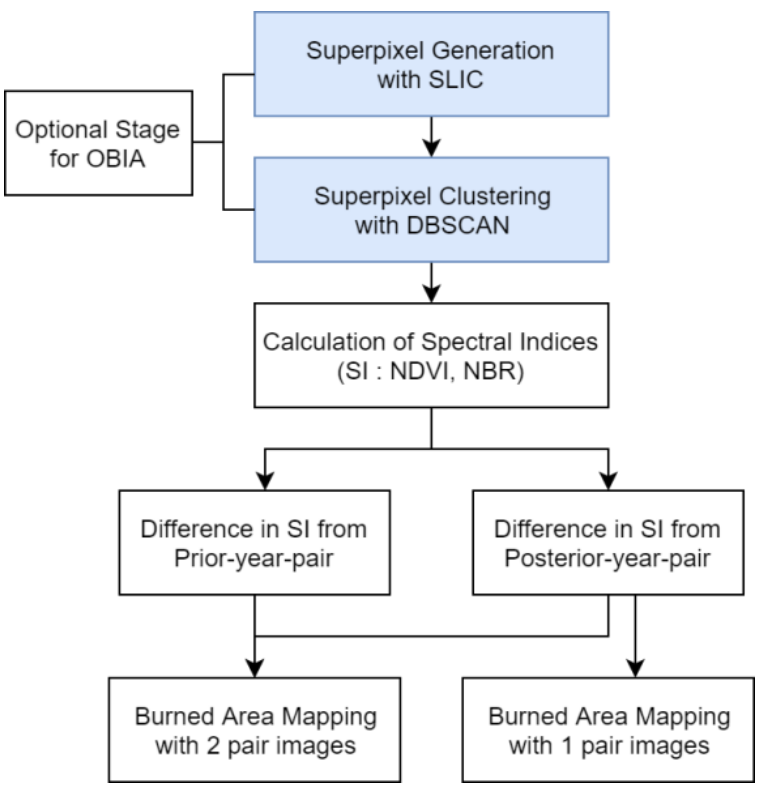

Figure 3. Flowchart of this study

\subsection{Superpixel Generation}

The SLIC technique, used to generate superpixel in this study, utilizes colour and spatial information of the image to efficiently generate uniform superpixels (Achanta et al., 2010). The reason for employing SLIC is mainly on the fact that it is based on RGB bands which have high spatial resolution and much less input parameter is required when compared with other conventionally used image segmentation methods. SLIC is also well recognized for outperforming the previous superpixel methods with high image segmentation quality (Achanta et al., 2012). High segmentation performance of SLIC is enabled by performing pixel clustering in CIELAB colour space, which was devised for perceptual colour space so that the differences in colours can be computed in Euclidean distances. In principle, SLIC performs local clustering in 5-dimensional space defined by L, a, b values from CIELAB colour space and $\mathrm{x}$, y pixel coordinates within $2 \mathrm{~S}$-by-2S (pixels) search region. As a parameter for the grid interval between initial cluster centers, $\mathrm{S}$ also implies the expected superpixel size from the desired number of superpixels. The only parameter required for SLIC is the desired number of superpixels from the image, and we tried to find out the optimal size of superpixel by comparing the ability to recall boundary of burned area for various superpixel sizes. SLIC was performed for discrete superpixel size of 10-by-10 to 35 -by-35 pixels. Then, for the superpixels which overlapped more than $50 \%$ of pixels with manuallydriven reference data were extracted as burned area. From Table 3 , smaller superpixels showed higher boundary adherence to the burned area as expected. Based on the statistics, we set the expected size of a single superpixel as 15 -by-15 pixels retaining omission and commission error for burned class to be less than $5 \%$ and overall accuracy to be over $99 \%$. For each image, SLIC was performed respectively without any integration between paired images.

\begin{tabular}{|c|c|c|c|}
\hline \multirow{2}{*}{$\begin{array}{c}\text { Superpixel } \\
\text { size }\end{array}$} & \multirow{2}{*}{$\begin{array}{c}\text { Overall } \\
\text { accuracy (\%) }\end{array}$} & $\begin{array}{c}\text { Omission } \\
\text { error (\%) }\end{array}$ & $\begin{array}{c}\text { Commission } \\
\text { error }(\%)\end{array}$ \\
\hline
\end{tabular}

\begin{tabular}{|c|c|c|c|}
\hline $10 \times 10$ & 99.260 & 2.490 & 4.268 \\
\hline $15 \times 15$ & 99.040 & 3.845 & 4.965 \\
\hline $20 \times 20$ & 98.798 & 4.540 & 6.433 \\
\hline $25 \times 25$ & 98.394 & 5.692 & 8.833 \\
\hline $30 \times 30$ & 98.152 & 6.595 & 10.075 \\
\hline $35 \times 35$ & 98.010 & 6.364 & 11.370 \\
\hline
\end{tabular}

Table 3. Overall accuracy, omission error, and commission error for burned class from the corresponding superpixel size

\subsection{Superpixel Clustering}

In the following steps, the produced superpixels were clustered into a certain size of objects for change detection. For superpixel clustering, DBSCAN (Ester et al., 1996; Kovesi) was employed to form clusters of superpixels, and mean Lab color distance (Equation 1) between the two adjacent superpixels was used as a clustering distance measure to determine the neighborhood of superpixels. The use of an adjacency matrix allows the DBSCAN to be efficient in determining the neighborhood of each superpixel.

\section{Lab Color Distance}

$$
=\sqrt{\left(\mathrm{L}_{1}-\mathrm{L}_{2}\right)^{2}+\left(\mathrm{a}_{1}-\mathrm{a}_{2}\right)^{2}+\left(\mathrm{b}_{1}-\mathrm{b}_{2}\right)^{2}}
$$

where

$\mathrm{L}=$ lightness

$\mathrm{a}=$ green-red color components

$\mathrm{b}=$ blue-yellow color components in CIELAB

(L, a, b with subscript number 1 and 2 corresponds to

$\mathrm{L}, \mathrm{a}, \mathrm{b}$ values of two adjacent superpixels.)

\subsection{Burned Area Detection}

To discriminate the burned region from unburned region, two commonly used SIs were employed, NDVI and NBR (Equation 2 and 3). By the application of the indices showing the status of vegetation, we set the wildfire-induced vegetation loss as the main changes to detect in this study. Considering the spatial resolution of spectral bands used to calculate the indices (B4, B8 : 10m, B12:20m), the final burned area mapping results from NDVI and NBR have a spatial resolution of $10 \mathrm{~m}$ and more than $10 \mathrm{~m}$ (between $10 \mathrm{~m}$ to $20 \mathrm{~m}$ ), respectively.

$$
\begin{gathered}
\mathrm{NDVI}=\frac{\mathrm{NIR}-\mathrm{Red}}{\mathrm{NIR}+\mathrm{Red}}=\frac{\mathrm{B} 8-\mathrm{B} 4}{\mathrm{~B} 8+\mathrm{B} 4} \\
\mathrm{NBR}=\frac{\mathrm{NIR}-\mathrm{SWIR} 2}{\mathrm{NIR}+\mathrm{SWIR} 2}=\frac{\mathrm{B} 8-\mathrm{B} 12}{\mathrm{~B} 8+\mathrm{B} 12}
\end{gathered}
$$

In OBIA, the changes were detected based on the final image segmentation results by calculating the features of the objects; in this case, mean NDVI and NBR within each object. First of all, by thresholding the mean NDVI images, objects with mean NDVI less than 0.1 were extracted as water mask. Then, the differences of NDVI and NBR (Equation 4 and 5) were calculated for each pair of images. The estimated dNDVI and dNBR values from 2019 image pair were thresholded with Otsu's thresholding (Otsu, 1979) for one pair multi-temporal image analysis. However, the vegetation differences captured by one pair image set may contain phenological changes as well as fire-induced changes. Therefore, to exclude these irrelevant changes with two pairs of image set, dNDVI and dNBR were also calculated from an additional image pair from 2018 and subtracted from the posterior-year-pair values before applying thresholding. 


$$
\begin{gathered}
\mathrm{dNDVI}=\left(\mathrm{NDVI}_{\text {prefire }}-\mathrm{NDVI}_{\text {postfire }}\right) \\
\mathrm{dNBR}=\left(\mathrm{NBR}_{\text {prefire }}-\mathrm{NBR}_{\text {postfire }}\right)
\end{gathered}
$$

For pixel-based analysis, the process so far was performed exactly the same way without image segmentation.

\section{EXPERIMENTAL RESULTS}

The point of this study is to verify the influence of multitemporal image analysis when integrated with both object and pixel-based image analysis. Parameter for image segmentation was chosen by optimal parameter selection and applied to each image separately. The proposed wildfire change detection method was applied to 1 pair or 2 pairs of high-resolution images from Sentinel-2 sensor. In doing so, NDVI and NBR were used as measures to detect the burned area. For evaluation of change detection performance, reference data was produced manually with visual interpretation.

From visual inspection (Figure 4 and 5), all the results showed considerably high discriminating power for the burned region. However, for the results from OBIA, two SIs behaved differently depending on the number of image pairs used to detect the burned area. While NDVI tends to miss some burned area with one pair of images and improved its change detection performance with two pairs of images, the results from NBR appeared to be deteriorated when two pairs of images were employed. It can also be verified with quantitative assessment results (Table 4 and 5), especially by omission error for the burned region of each case. This trend was also identified in the pixel-based analysis. It implies that the additional annual information can only improve the fire-induced change detection quality when carefully considering the spectral responses from both burned and unburned region.

The accuracy of change detection was assessed for eight cases depending on the image processing unit, the number of image pairs, and SIs (Table 4 and 5). From Table 4, pixel-based image analysis with 1-pair of NBR images showed the highest overall accuracy of $98.507 \%$, but it includes removal of the noisy pixels, and without such removal, the accuracy dropped to $97.960 \%$. It is still relatively high overall accuracy, yet it requires human intervention in determining the removable size of pixels. Among two SIs, NBR slightly outperformed NDVI for detecting the vegetation changes from the wildfire. However, the spatial resolution of SWIR band required for calculation of NBR is usually lower than red or NIR band, and it limits the applicability of NBR to more detailed analysis with higher spatial resolution images. In that sense, NDVI results have the advantage of high spatial resolution, and then, the critical factor for utilizing NDVI will fall into the acquisition of multitemporal images.

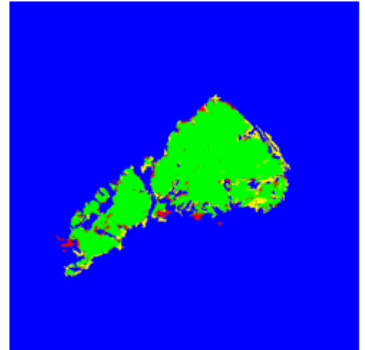

(a)

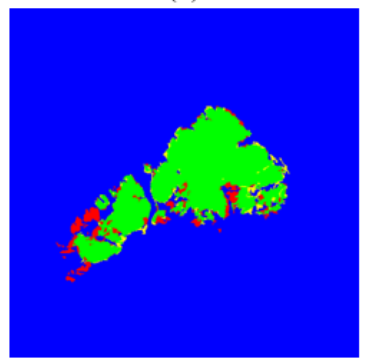

(c)

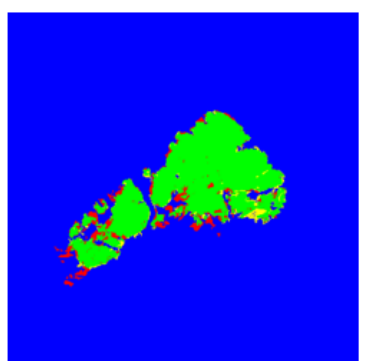

(b)

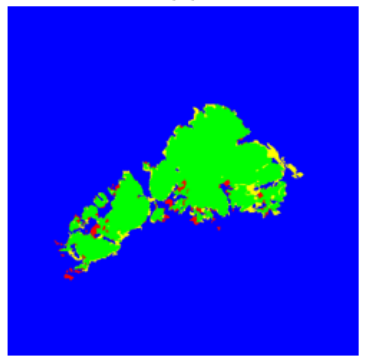

(d)

\begin{tabular}{|lccc|}
\hline & True Positive & True Negative \\
$\square$ & False Positive & False Negative \\
\hline
\end{tabular}

Figure 4. Burned area mapping results from OBIA with two pairs of images; (a) NDVI, (b) NBR; with one pair of images (c) NDVI, (d) NBR

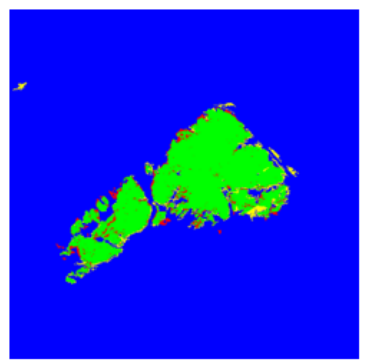

(a)

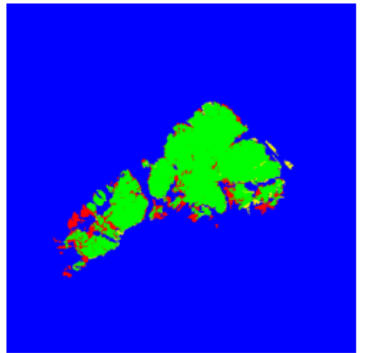

(c)

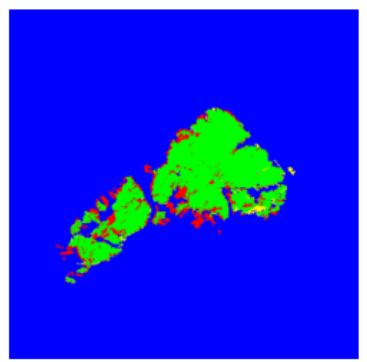

(b)

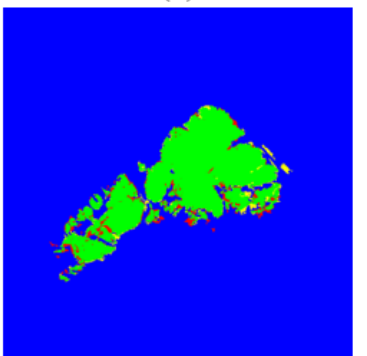

(d)

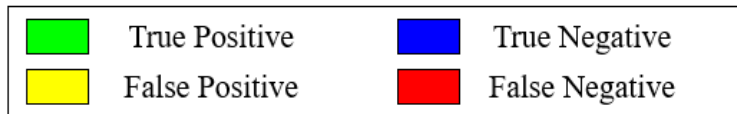

Figure 5. Burned area mapping results from pixel-based image analysis with two pairs of images; (a) NDVI, (b) NBR; with one pair of images (c) NDVI, (d) NBR 


\begin{tabular}{|c|c|c|c|c|c|c|c|}
\hline \multirow{2}{*}{ Method } & \multirow{2}{*}{$\begin{array}{l}\text { Number of } \\
\text { image pairs }\end{array}$} & \multirow{2}{*}{$\begin{array}{c}\text { Spectral } \\
\text { index }\end{array}$} & \multirow{2}{*}{$\begin{array}{c}\text { Overall accuracy } \\
(\%)\end{array}$} & \multicolumn{2}{|c|}{ Producer's accuracy $(\%)$} & \multicolumn{2}{|c|}{ User's accuracy (\%) } \\
\hline & & & & Unburned & Burned & Unburned & Burned \\
\hline \multirow{4}{*}{$\begin{array}{c}\text { Object- } \\
\text { based }\end{array}$} & \multirow{2}{*}{2 pair } & NDVI & 98.039 & 98.568 & 93.679 & 99.227 & 88.821 \\
\hline & & NBR & 98.085 & 99.327 & 87.863 & 98.538 & 94.065 \\
\hline & \multirow{2}{*}{1 pair } & NDVI & 97.946 & 99.278 & 86.985 & 98.433 & 93.600 \\
\hline & & NBR & 98.198 & 98.662 & 94.375 & 99.312 & 89.547 \\
\hline \multirow{4}{*}{$\begin{array}{l}\text { Pixel- } \\
\text { based }\end{array}$} & \multirow{2}{*}{2 pair } & NDVI & 98.189 & 98.935 & 92.043 & 99.033 & 91.302 \\
\hline & & NBR & 97.729 & 99.633 & 82.056 & 97.859 & 96.448 \\
\hline & \multirow{2}{*}{1 pair } & NDVI & 97.925 & 99.559 & 84.468 & 98.140 & 95.881 \\
\hline & & NBR & 98.507 & 99.324 & 91.781 & 99.005 & 94.279 \\
\hline
\end{tabular}

Table 4. Accuracy assessment (Overall accuracy, producer's accuracy, user's accuracy)

\begin{tabular}{|c|c|c|c|c|c|c|c|}
\hline \multirow{2}{*}{ Method } & \multirow{2}{*}{$\begin{array}{l}\text { Number of } \\
\text { image pairs }\end{array}$} & \multirow{2}{*}{$\begin{array}{l}\text { Spectral } \\
\text { index }\end{array}$} & \multicolumn{2}{|c|}{ Commission error $(\%)$} & \multicolumn{2}{|c|}{ Omission error (\%) } & \multirow{2}{*}{$\begin{array}{c}\text { Kappa } \\
\text { coefficient }\end{array}$} \\
\hline & & & Unburned & Burned & Unburned & Burned & \\
\hline \multirow{4}{*}{$\begin{array}{c}\text { Object- } \\
\text { based }\end{array}$} & \multirow{2}{*}{2 pair } & NDVI & 0.773 & 11.179 & 1.432 & 6.321 & 0.9008 \\
\hline & & NBR & 1.462 & 5.935 & 0.673 & 12.137 & 0.8979 \\
\hline & \multirow{2}{*}{1 pair } & NDVI & 1.567 & 6.400 & 0.722 & 13.015 & 0.8903 \\
\hline & & NBR & 0.688 & 10.453 & 1.338 & 5.625 & 0.9088 \\
\hline \multirow{4}{*}{$\begin{array}{l}\text { Pixel- } \\
\text { based }\end{array}$} & \multirow{2}{*}{2 pair } & NDVI & 0.967 & 8.698 & 1.065 & 7.957 & 0.9065 \\
\hline & & NBR & 2.141 & 3.552 & 0.367 & 17.944 & 0.8742 \\
\hline & \multirow{2}{*}{1 pair } & NDVI & 1.860 & 4.119 & 0.441 & 15.532 & 0.8866 \\
\hline & & NBR & 0.995 & 5.721 & 0.676 & 8.219 & 0.9218 \\
\hline
\end{tabular}

Table 5. Accuracy assessment (Commission error, omission error, Kappa coefficient)

\section{CONCLUSION}

Object and pixel-based wildfire damage assessment were performed for Sentinel-2 satellite images to address the influence of employing multi-temporal images. To detect the changed regions, NDVI and NBR indices were obtained from red and NIR, and NIR and SWIR band respectively and provide discriminative responses for the burned region. For OBIA, image segmentation was performed using SLIC superpixels to cluster pixels into a certain size of objects. NDVI and NIR were calculated for both object and pixel-based analysis as measures to detect vegetation loss and then by thresholding each value, and the final burned area mapping results were obtained. The change detection results generally showed similar trends with reference data, well representing the boundaries of the burned region. However, two spectral indices showed quite different behavior depending on the number of image pairs. With NDVI, two pairs of images improved the accuracy by excluding the unwanted vegetation changes (e.g. phenological changes) from fire-induced vegetation changes.

Meanwhile, NBR tends to be more accurate when applied to the only one pair of images implying differences in spectral responses may affect the change detection by eliminating irrelevant changes or even deleting the fire-induced changes as well. Therefore, to enhance the accuracy of the results, further investigation should be carried out on the relationship between spectral responses for coniferous forests in South Korea with a substantial amount of case studies. Besides, for the comparison of object and pixel-based image analysis, a relatively simple change detection method was applied for providing similar conditions in this study. Thus the integration of additional features such as textural, contextual and morphological information will enable more accurate burned are mapping to be possible.

With the stable full operation of Sentinel-2A and -2B, Sentinel2 satellites offer favorable opportunities for wildfire damage analysis as they are collecting high temporal resolution (5 days) data. Furthermore, based on data interoperability of Sentinel-2 with other spaceborne image sensing platforms, the accuracy of disaster damage assessment will be even improved along with near-daily monitoring of earth surface.

\section{ACKNOWLEDGEMENTS}

This research was supported by a grant (2019-MOIS32-015) of Disaster-Safety Industry Promotion Program funded by Ministry of Interior and Safety (MOIS, Korea).

\section{REFERENCES}

Achanta, R., Shaji, A., Smith, K., Lucchi, A., Fua, P., Süsstrunk, S., 2010. SLIC Superpixels. EPFL Technical Report 149300

Achanta, R., Shaji, A., Smith, K., Lucchi, A., Fua, P., Süsstrunk, S., 2012. SLIC superpixels compared to state-of-theart superpixel methods. IEEE Transactions on Pattern Analysis and Machine Intelligence, 34(11), 2274-2282.

Aggarwal, N., Srivastava, M., Dutta, M., 2016: Comparative analysis of pixel-based and object-based classification of high resolution remote sensing images - a review. International Journal of Engineering Trends and Technology, 38(1), 5-11.

Blaschke, T., Lang, S., Lorup, E., Strobl, J., Zeil, P., 2000: Object-oriented image processing in an integrated GIS/remote sensing environment and perspectives for environmental applications. Environmental Information for Planning, Politics and the Public, 2, 555-570.

ESA, 2015. SENTINEL-2 User Handbook. Accessed 19 July 2019.https://sentinels.copernicus.eu/documents/247904/685211/ Sentinel-2_User_Handbook/ 
Ester, M., Kriegel, H. P., Sander, J., Xu, X., 1996. A densitybased algorithm for discovering clusters in large spatial databases with noise. In: International Conference on Knowledge Discovery and Data Mining (KDD-96), 2-4 August, 1996, Portland, Oregon, USA, 96(34), pp. 226-231.

Huang, H., Roy, D., Boschetti, L., Zhang, H., Yan, L., Kumar, S., Gomez-Dans, J., Li, J., 2016: Separability analysis of Sentinel-2A multi-spectral instrument (MSI) data for burned area discrimination. Remote Sensing, 8(10), 873.

Hussain, M., Chen, D., Cheng, A., Wei, H., Stanley, D., 2013: Change detection from remotely sensed images: From pixelbased to object-based approaches. ISPRS Journal of Photogrammetry and Remote Sensing, 80, 91-106.

Kovesi P., MATLAB and Octave Functions for Computer Vision and Image Processing. Accessed 20 July 2019. http://www.peterkovesi.com/matlabfns/

Lentile, L. B., Holden, Z. A., Smith, A. M., Falkowski, M. J., Hudak, A. T., Morgan, P., Lewis, S. A., Gessler, P. E., Benson, N. C., 2006: Remote sensing techniques to assess active fire characteristics and post-fire effects. International Journal of Wildland Fire, 15(3), 319-345.

Liu, Y., Stanturf, J., Goodrick, S., 2010: Trends in global wildfire potential in a changing climate. Forest Ecology and Management, 259(4), 685-697.

Mallinis, G., Mitsopoulos, I., Chrysafi, I., 2018: Evaluating and comparing Sentinel 2A and Landsat-8 Operational Land Imager (OLI) spectral indices for estimating fire severity in a Mediterranean pine ecosystem of Greece. GIScience \& Remote Sensing 55(1), 1-18.

Martinis, S., Caspard, M., Plank, S., Clandillon, S., Haouet, S., 2017. Mapping burn scars, fire severity and soil erosion susceptibility in Southern France using multisensoral satellite data, In: IEEE International Geoscience and Remote Sensing Symposium (IGARSS), 23-28 July, 2017, Fort Worth, Texas, USA, pp. 1099-1102.

Navarro, G., Caballero, I., Silva, G., Parra, P. C., Vázquez, Á., Caldeira, R., 2017: Evaluation of forest fire on Madeira Island using Sentinel-2A MSI imagery. International Journal of Applied Earth Observation and Geoinformation, 58, 97-106.

Neubert, P., Protzel, P., 2012. Superpixel benchmark and comparison. In: Forum Bildverarbeitung, 6.

Otsu, N., 1979: A threshold selection method from gray-level histograms. IEEE Transactions on Systems, Man, and Cybernetics, 9(1), 62-66. 